\title{
Miniaturized Antenna with Combination of Meander and Square Spiral Slots for Biomedical Applications
}

\author{
R.Brinda \\ Electronics and Communication \\ Mepco Schlenk Engineering College \\ Sivakasi, India
}

\author{
S. Sara Preethy \\ Electronics and Communication \\ Mepco Schlenk Engineering College \\ Sivakasi, India
}

\begin{abstract}
The implantable medical devices (IMDs) are one of the most important advanced healthcare systems. Nowadays, the devices which are designed to monitor physiological data from inside the human body have great promises to provide major contributions to disease prevention, diagnosis and therapy thus reducing hospitalization terms and improving the patients' quality of life.

It is recognized that modern wireless technology will play an important role in the biomedical application. Essential elements of implantable devices are antenna embedded in system contains Biosensors and interface circuits, which enable the exchange of data between implantable devices and external environment. In this paper, the implantable slot antenna which operates at MICS band $(402-405 \mathrm{MHz})$ has been designed. This implantable antenna is a combination of meander slots and square spiral slots have been embedded for effective size reduction at a fixed frequency operation. Compared to traditional planar inverted-F antennas (PIFAs), the proposed antenna has advantages of good size reduction and also being easy to be optimized to the necessary resonance frequency. This design would fulfill the requirements of biocompatibility, miniaturization, patient safety, and high-quality communication with exterior equipment.
\end{abstract}

\section{Keywords}

Implantable slot antenna, Medical Implant Communications Service (MICS) band, Slot antenna, Small antenna.

\section{INTRODUCTION}

The development of Integrated Circuits (ICs), over the years, has facilitated the evolution of complex and highly integrated small medical devices. The increasing demand for noninvasive surgical operations has made the use of Implantable Medical Devices (IMDs) as part of medical procedures that is highly attractive. Implantable medical devices have wide variety of applications according to their functions and it is categorized as following, the first category includes all those devices used to diagnose various diseases. These IMDs in addition to their communication system with the external environment include some sensors that interact with the human body to measure the necessary physiological data. This category includes Microsystems implanted within the human body (i.e. temperature monitors, electrocardiograms ECG, blood-glucose sensors etc.) to monitor important biosignals. The second category includes implantable devices used as stimulators. Stimulators receive information from an external unit which is usually managed by doctors and used to stimulate specific nerves. Such devices are pacemakers and cardioverter defibrillators, Functional Electrical Stimulators (FES), cochlear and retinal implants.
Implantable communication devices have great attention in the medical field to remotely monitor various vital signs of patients. This is mainly used for monitoring, recording and measuring of a living organism's basic physiological function such as heart rate, muscle activity etc... Nowadays implantable antennas for medical applications have become a research focus [1]-[21]. In [1], microstrip patch antenna for communication with MICS band is designed. The effects of shape, length, size, location of feed point and ground point, substrate and superstrate materials and their thicknesses are evaluated in this paper. In [2], a broadband nonsuperstrate implantable coplanar waveguide (CPW)-fed monopole antenna with bandwidth of $33.5 \%$ was developed on a ceramic substrate with high dielectric constant. A single-fed miniaturized hybrid patch/slot implantable antenna in [3] embedding the meander slot and six open slots in the ground, for good size reduction and it is optimized to necessary resonance frequency. PIFA at high frequency achieve enhanced gain, reduced SAR values, increased maximum allowable net input power level and good quality of communication with exterior equipment in [4].

There are many techniques available to get miniaturized antenna such as the addition of ground pins in the patch, high dielectric constant substrate materials and transforming the conductor shape into a spiral etc... There are many frequency bands such as MICS, ISM and Med Radio band approved for medical implants. Among that the Medical Implant Communications Service (MICS band $402-405 \mathrm{MHz}$ ) is most commonly used for Biotelemetry applications. This band could be used for long-term medical implants, and therefore it has less interference among the various ambient radio waves.

In this paper, the implantable slot antenna which operates at MICS band (402-405 MHz) has been designed for biotelemetry devices as shown in Fig.1. By embedding a combination of meander slots and square spiral slots on the patch properly, the current path on the patch can be lengthened thus lowering the antenna's resonant frequency for effective size reduction. Compared to traditional planar inverted-F antennas (PIFAs), the proposed antenna has advantages of good size reduction at a fixed frequency operation and also being easy to be optimized to the necessary resonance frequency.

Meanwhile, the open end slots and via hole introduction in the patch could extend the current path to enhance the size reduction. Thus the compact implantable slot antenna is realized using ADS simulation software.

\section{MINIATURIZATION TECHNIQUE}

The following techniques in [5] are followed in designing implantable slot antenna operated at MICS band for the various biomedical applications, 


\subsection{Meander Type Slot Antenna}

To achieve lower resonant frequency (MICS band), meander type slot antenna are used. This will increase the efficiency of antenna by reducing dissipation. The idea of using meander type slot is to fold the conductors back and forth to make the overall antenna shorter.

\subsection{Slotted Spiral Antenna}

Slotted spiral antenna is a series inductive element. It is used at the end of the slot antenna, which could short-circuit any termination. To overcome this problem, a lumped inductor could be physically realized by a compact short-circuited slotted spiral.

\subsection{Via Hole}

Via hole is used for lengthening the current path in patch as well as to excite more modes and tune the impedance matching to achieve lower resonance frequency.

\subsection{Antenna Feed Location}

A microstrip transmission line is used to feed the center of the slot antenna. The choice of the microstrip feed as compared to a coaxial line is based on the ease of fabrication and stability. This feed structure is easier to tune. The coplanar waveguide (CPW) can be used to feed the antenna providing the ease of fabrication, whereas it is more difficult to tune.

\subsection{Patch Stacking}

Patch stacking method is to stack the conductor one above the other to make the overall size of the antenna shorter. The height of the substrate should be high to achieve lower resonant frequency.

\subsection{Non uniform spiral and meander width}

The non-uniform slot width antenna shows smaller in size and wider in bandwidth than the previous uniform slot antenna. Variable metal width is the potential structure for achieving high-Q performance by suppressing eddy current losses in the inner turns.

\subsection{Resonant circuit}

Resonant circuit is used to select a range of frequencies from the ambient radio waves. This circuit is often referred to as a tuned circuit.

\section{ANTENNA DESIGN}

Miniaturized implantable slot antenna are designed to operated at MICS band (402-405 MHz) by embedding combination of meander \& spiral slot in the patch. For effective size reduction of the antenna, some of the miniaturization techniques like use of high permittivity dielectric substrate material, meander and spiral slot combinations are used to lengthen the current flow path on the radiating patch, addition of shorting pins and patch stacking are used to obtain lower resonant frequency.

The proposed implantable slot antenna is as shown in Fig.1. This antenna is built by using Rogers \& Duroid substrate with specific dielectric constant and thickness of the substrate.

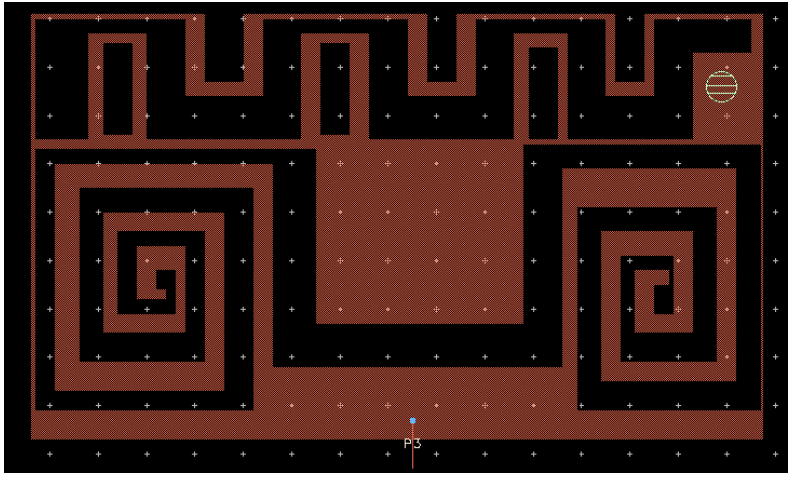

Fig.1.Proposed Implantable slot Antenna Profile

The implantable slot antenna is initially designed as patch antenna which operated at $403 \mathrm{MHz}$. Since it occupies large volume, meander type slot antenna is used for designing miniaturized implantable slot antenna.

Then the proposed implantable slot antenna consists of two patch elements stacked one above the other. In order to get miniaturized antenna, via hole is introduced to lengthen the current flow path to achieve lower resonance frequency bandwidth (402-405 MHz MICS band) for biomedical applications. This complete antenna is designed using Advanced Design System (ADS) using Rogers \& Duroid substrate with $1.27 \& 0.2 \mathrm{~mm}$ and a relative permittivity of 10.8. This antenna is designed to operate in the MICS band (402-405MHz).

This proposed antenna has measured compact dimensions of $8.8 \times 15.2 \times 1.27 \mathrm{~mm}$. In order to achieve lower resonance frequency, the feed point is given at the centre, so that the current flows through the slot antenna are not confined to the edges of the slot but rather spread over the sheet. Further open-end slots with uniform width of $0.2 \mathrm{~mm}$ were cut in the proposed antenna structure. So the bandwidth at the lower band is broadened significantly. A shorting pin of diameter $0.2 \mathrm{~mm}$ was used to increase the electrical length and it minimizes the antenna design. By adjusting meander and spiral slot width, MICS band is achieved by suppressing the eddy current loss in the inner turns of the spiral to achieve better Q Performance. The final simulated result obtained for this design is as shown in Fig.2

The Return loss should be S11 <-10 db. To get good return loss, the lumped component circuit is implemented in the schematic window by including the antenna structure from layout design in the component library using ADS simulation software.

Thus miniaturized implantable slot antenna is designed to operate at MICS band for various biomedical applications. By comparing with the previous design of implantable slot antenna, the proposed design which operate at MICS Band achieve reduction in size is shown in Table.1. The main advantage of the proposed antenna includes miniaturization which is used for many Biotelemetry devices.

The radiation pattern of the designed implantable slot antenna is shown in Fig.4. The radiation pattern covers the frequency range of 402-405 MHz (MICS BAND). 


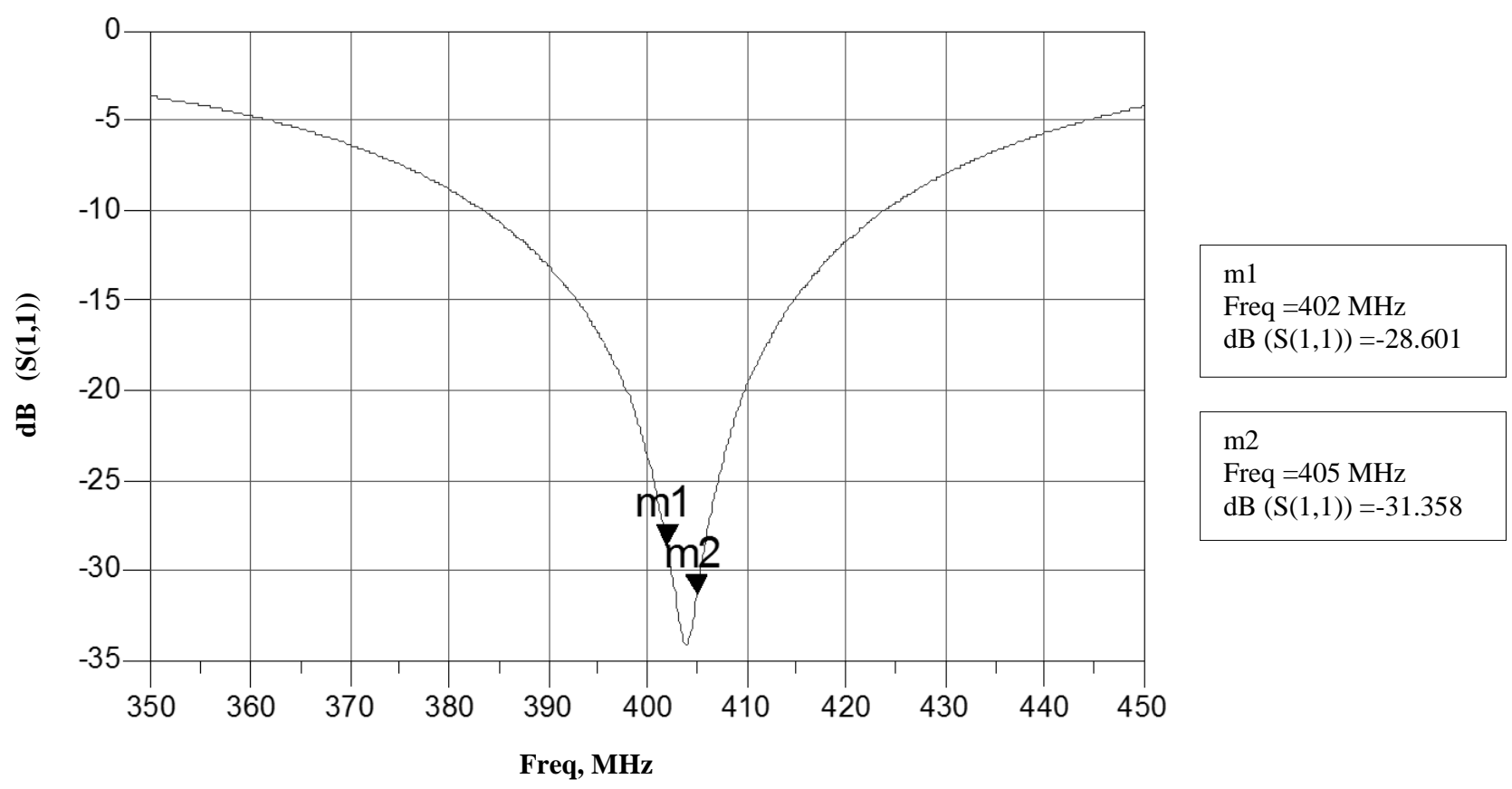

Fig.2. Return loss for the implantable antenna operate at MICS band

Table.1.Miniaturization in the proposed implantable slot antenna

\begin{tabular}{|c|c|c|c|c|c|c|}
\hline \multirow{2}{*}{$\begin{array}{l}\text { Bands } \\
\text { (MHz) }\end{array}$} & \multirow{2}{*}{$\begin{array}{l}\text { Substrate } \\
\text { shape }\end{array}$} & \multicolumn{4}{|c|}{ Miniaturization technique } & \multirow{2}{*}{$\begin{array}{l}\text { Volume } \\
(\mathrm{mm3})\end{array}$} \\
\hline & & Dielectric material & Patch shape & $\begin{array}{l}\text { Shorting } \\
\text { pins }\end{array}$ & $\begin{array}{l}\text { Patch } \\
\text { stacking }\end{array}$ & \\
\hline $\begin{array}{l}\text { 402-405 } \\
{[3]}\end{array}$ & Rectangle & $\begin{array}{l}2 \text { layer Rogers } \\
3010\end{array}$ & Meander slot & Yes & Yes & 203.2 \\
\hline $402-405$ & Rectangle & Rogers/ Duroid & $\begin{array}{l}\text { Meander\& } \\
\text { spiral slot }\end{array}$ & Yes & Yes & 196 \\
\hline
\end{tabular}

MICS band (402-405) MHz. The proposed antenna has measured compact dimensions of $8.8 \times 15.2 \times 1.27 \mathrm{~mm}$. This implantable slot antenna has been developed for biotelemetry
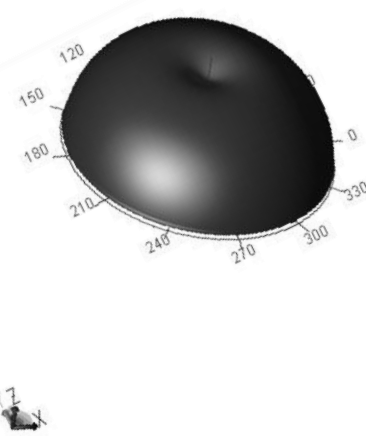

Fig.4. 3D visualization of radiation pattern for implantable slot antenna

\section{CONCLUSION}

Miniaturized implantable slot antenna by embedding combination of meander \& spiral slot is designed to operate at applications with a wide lower band. A slotted path has been used to improve the bandwidth at the lower band without increasing the volume of the antenna.

In the proposed antenna, the required result of bandwidth from $402 \mathrm{MHZ}$ to $405 \mathrm{MHZ}$ frequency is obtained. The realized gain is around $-28 \mathrm{db}$ and directivity $5.2 \mathrm{db}$ are obtained. The use of lumped circuit with the antenna decreases the return loss below $-11 \mathrm{db}$. For effective size reduction of the antenna operating at lower resonance frequency, some of the miniaturization techniques like use of high permittivity dielectric substrate material, meander and spiral slot to lengthen the current flow path on the radiating patch, addition of shorting pins and patch stacking are used.

The proposed meander spiral slot antenna is well matched at $402 \mathrm{MHz}$ with $-28.6 \mathrm{~dB}$ reflection coefficient. The fractional bandwidth of the antenna was calculated to be $17 \%$. The Radiated Power is enough for communication with outside of the body that preserve patient safety and biocompatibility. However, future work could be fulfilled to improve the Radiation Efficiency. 


\section{REFERENCES}

[1] P. Soontornpipit, C. M. Furse, and Y. C. Chung, "Design of implantable microstrip antennas for communication with medical implants," IEEE Trans. Microw. Theory Tech., vol. 52, no. 8, pp. 1944-1951, Aug. 2004.

[2] T. F. Chien, C.-M. Cheng, H.-C. Yang, J.-W. Jiang and C.-H. Luo,"Development of nonsuperstrate

Implantable low-profile CPW-fed ceramic antennas," IEEE Antennas Wireless Propag. Lett, vol. 9, pp.599$602,2010$.

[3] Changrong Liu, Yong-Xin Guo, Shaoqiu Xiao, "A Hybrid Patch/Slot Implantable Antenna for Biotelemetry Devices," Antennas Propag. Mag. IEEE, vol. 11, Jun. 2012.

[4] A. Kiourti and K. S. Nikita, "Miniature Scalp Implantable Antennas for Telemetry in the MICS and ISM Bands: Design, Safety Considerations and Link Budget Analysis," IEEE Trans. Antennas Propag.

[5] A. Kiourti and K. S. Nikita, "A Review of Implantable Patch Antennas for Biomedical Telemetry: Challenges and Solutions," Antennas Propag. Mag. IEEE, vol. 54, no. 3, pp. 210 - 228, Jun. 2012.

[6] C.-M. Lee, T.-C. Yo, C.-H. Luo, C.-H. Tu and Y.-Z. Juang, "Compact broadband stacked implantable antenna for biotelemetry with medical devices." Electron. Lett. vol. 43, no. 12, pp. 660-662, Jun. 7, 2007.

[7] M. Meyers, P. Chen, A. Lin, and Y. Seki, "Biological materials: Structure and mechanical properties," Prog. Mater. Sci., no. 53, pp. 201-206, 2008.

[8] T. Karacolak, A. Hood, and E. Topsakal, "Design of a dual-band implantable antenna and development of skin mimicking gels for continuous glucose monitoring," IEEE Trans. Microw. Theory Tech., vol. 56, no. 4, pp. 1001-1008, Apr. 2008.

[9] T. Karacolak, R. Cooper, and E. Topsakal, "Electrical properties of rat skin and design of implantable antennas for medical wireless telemetry," IEEE Trans. Antennas Propag., vol. 57, no. 9, pp. 2806-2812, Sep. 2009.

[10] W.-C. Liu, S.-H. Chen and C.-M. Wu, "Bandwidth enhancement and size reduction of an implantable PIFA antenna for biotelemetry devices," Microw. Opt. Technol. Lett., vol. 51, pp. 755-757, Mar. 2009.

[11] W. Xia, K. Saito, M. Takahashi, and K. Ito, "Performances of an implanted cavity slot antenna embedded in the human arm," IEEE Trans. Antennas Propag., vol. 57, no. 4, pp. 894-899, Apr. 2009.

[12] S. Stupp and P. Braun, "Molecular Manipulation of Microstructures: Biomaterials, Ceramics, and Semiconductors," Science, no. 277, pp. 1242-1248, 1997.

[13] T. Karacolak, R. Cooper, J. Butler, S. Fisher, and E. Topsakal, "In vivo verification of implantable antennas using rats as model animal," IEEE Antennas Wireless Propag. Lett. vol. 9, pp. 334-337, 2010.
[14] C. J. Sanchez Fernandez, O. Quevedo-Teruel, J. Requena-Carrión, L. Inclán-Sánchez, and E. RajoIglesias, "Dual-band microstrip patch antenna based on short-circuited ring and spiral resonators for implantable medical devices," Microw., Antennas Propag., vol. 4, no. 8, pp. 1048-1055, 2010.

[15] A. Kiourti, M. Tsakalakis, and K. S. Nikita, "Parametric Study and Design of Implantable PIFAs for Wireless Biotelemetry," Proc. 2nd Icst Int. Conf Wirel. Mob. Common. Health. Mobihealth 2012 Kos Isl. Greece, Oct. 2011.

[16] A. Kiourti, K. S. Nikita, and M. Christopoulou, "Performance of a Novel Miniature Antenna Implanted in the Human Head for Wireless Biotelemetry," IEEE Int. Symp. Antennas Propag. Spokane Wash., Jul. 2011.

[17] P. D. Bradley, "Wireless Medical Implant Technology - Recent Advances and Future Developments," Esscirc 2011 Proc., pp. 37 - 41, 2011.

[18] A. K. Skriverviky and F. Merli, "Design strategies for implantable antennas," Antennas Propag. Conf. Lapc 2011 Loughb., pp. 1 - 5, Nov. 2011

[19] F. Merli, "Implantable Antennas for Biomedical Applications," Ecole Polytechnique Federale De Lausanne, 2011

[20] IEEE, "Standard for Safety Levels with Respect to Human Exposure to Radio Frequency Electromagnetic Fields, $3 \mathrm{kHz}$ to $300 \mathrm{GHz}$," 2005.

[21] A. Kumar, A. Srivastava, I. Galaev, and B Mattiasson, "Smart polymers: Physical forms and bioengineering applications," Prog. Polym. Sci., vol. 32 , no. 10 , pp. $1205-1237,2007$. 